Orange Journal / Volumen 2 Número 3/ Enero - Julio 2020

DOI: https://doi.org/10.46502/issn.2710-995X/2020.3.03

\title{
Determinación de la actividad antidedrepanocítica de nanopartículas cargadas con vainillina mediante Resonancia Magnética Nuclear
}

Antidrepanocytic activity determination of nanoparticles loaded with vanillin by Nuclear Magnetic Resonance

Recibido: 6 de enero de $2021 \quad$ Aceptado: 15 febrero de 2021

Escrito por:

Annarli Olivia Rodriguez Ferreiro ${ }^{8}$ https://orcid.org/0000-0002-3483-2804

Erich Hidalgo Reyes 9 https://orcid.org/0000-0002-4310-2109

Roger Rivero Labrada ${ }^{10}$ https://orcid.org/0000-0003-0372-3338

Emilia Ortiz Beatón ${ }^{11}$ https://orcid.org/0000-0002-4527-3809

Rubén López Noa ${ }^{12}$ https://orcid.org/0000-0001-9315-1729

\section{Resumen}

En la presente investigación se simula la actividad antidrepanocitaria en muestras biológicas (sangre total) frente a nanopartículas sólidas lipídicas cargadas con vainillina (NSL- V) a través de las mediciones de los tiempos de relajación magnética de las moléculas de hidrógeno. Para este fin se empleó sangre proveniente del Banco de Sangre del Hospital "Dr. Antonio María Béguez César" perteneciente a pacientes con Anemia Drepanocítica (AD) y se preparó un grupo control y tres grupos experimentales. Grupo 1 (G1): $300 \mu \mathrm{l}$ de sangre total SS. Grupo 2: $150 \mu \mathrm{l}$ de sangre total SS y $150 \mu 1 \mathrm{de}$ formulación de NSL con vainillina. Grupo 3 (G3): $150 \mu 1$ de sangre total SS y $150 \mu 1$ de formulación de vainillina. Grupo 4 (G4): $150 \mu \mathrm{l}$ de sangre total SS y $150 \mu \mathrm{l}$ de formulación de NSL) y cada uno se realizó por triplicado. Se obtuvieron nanopartículas sólidas lipídicas con valores de eficiencia de encapsulación superiores al 50\%, lo que demuestra una adecuada incorporación de vainillina en estos nanosistemas. Mediante el empleo de la técnica RMN se demostró que la vainillina encapsulada en NSL aumenta

\footnotetext{
${ }^{8}$ Departamento de Ingeniería Biomédica, Facultad de Ingeniería en Telecomunicaciones, Informática y Biomédica, Universidad de Oriente, Santiago de Cuba, Cuba.

9 Departamento de Farmacoepidemiología. Dirección municipal de Níquero. Granma. Cuba.

${ }^{10}$ Departamento de Ingeniería Biomédica, Facultad de Ingeniería en Telecomunicaciones, Informática y Biomédica, Universidad de Oriente, Santiago de Cuba, Cuba.

${ }^{11}$ Departamento de Ingeniería Biomédica, Facultad de Ingeniería en Telecomunicaciones, Informática y Biomédica, Universidad de Oriente, Santiago de Cuba, Cuba.

12 Departamento de Ingeniería Biomédica, Facultad de Ingeniería en Telecomunicaciones, Informática y Biomédica, Universidad de Oriente, Santiago de Cuba, Cuba.
} 


\title{
ORANGE JOURNAL
}

los valores del tiempo de demora (Td), lo que es indicativo de la inhibición de la formación de los polímeros de $\mathrm{HbS}$ en los eritrocitos falciformes avalando el uso de nanopartículas sólidas lipídicas como vehículos para aumentar la eficacia de la vainillina en el tratamiento de la Anemia Drepanocítica.

Palabras clave: Anemia Drepanocítica, vainillina, nanopartículas sólidas lipídicas, Resonancia Magnética Nuclear, tiempo de demora.

\begin{abstract}
The antidrepanocity activity on biological samples (whole blood) of solid lipid nanoparticles doped with vanillin (NSL-V) was assessed by measurements the magnetic relaxation of the hydrogen molecules. With this purpose, blood belonging to patients with sickle cell anemia offered by the blood bank of Hospital "Dr. Antonio María Béguez César". Three experimental groups plus the control were designed. Group 1: $300 \mu \mathrm{L}$ of total blood SS. Group 2: $150 \mu \mathrm{L}$ of total blood SS plus $150 \mu \mathrm{L}$ of formulation NSL with vanillin. Group 3: $150 \mu \mathrm{l}$ of total blood SS plus $150 \mu \mathrm{l}$ of formulation vanillin. Group 4 (G4): $150 \mu 1$ of total blood SS plus $150 \mu 1$ of formulation NSL. Solid lipid nanoparticles with high encapsulation efficiency where obtained (over 50\%), demonstrating the encapsulation capacity of the procedure employed. Using the NMR technique, it was possible to define that NSL-V increases the Td values, which is indicative of the HbS polymerization inhibition process. These evidences support the potential of NSL-V on the treatment of the cell sickle anemia disease.
\end{abstract}

Key Words: Sickle Cell Anemia, vanillin, solid lipid nanoparticles, Nuclear Magnetic Resonance, time delay.

\section{Introducción}

La Anemia Drepanocítica (AD), Drepanocitemia o Sicklemia es una enfermedad que predomina en la raza negra y se caracteriza porque los eritrocitos adquieren forma de hoz debido a la polimerización de la hemoglobina S desoxigenada (dHbS). (Nigen y Manning, 1977), (Beddell et al., 1979). Los glóbulos rojos falciformes ocluyen los vasos sanguíneos y son propensos a la hemólisis lo que induce crisis de dolor intenso, isquemias orgánicas y otras complicaciones sistémicas (Beddell et al., 1979).

Es la anemia hemolítica de carácter genético más frecuente en el mundo y está considerada como un problema de la salud pública en muchos países. En Cuba, se calcula que el número de portadores es de alrededor de 300 000, y el número de enfermos aproximadamente de 4000, para una incidencia del estado de portador del 3,08\% en la población general y del $6 \%$ en el color de piel negra y mestiza (Morejón et al., 2019).

En la actualidad no se cuenta con un tratamiento seguro y eficaz para erradicar la AD, son varios los abordajes terapéuticos que se emplean para disminuir las crisis y aumentar la esperanza de vida de los pacientes. El empleo de la nanotecnología como herramienta para la solución de este problema de salud mediante la encapsulación de compuestos naturales como la vainillina pudiera ser una alternativa terapéutica, ya que diversos 
estudios han demostrado que la vainillina posee la capacidad de inhibir el proceso de polimerización de la hemoglobina S (Cabal et al., 1998).

Los nanosistemas de liberación controlada de fármacos tienen propiedades que favorecen la protección, absorción y dirección de fármacos en el organismo (Lu et al., 2014). Se fabrican a partir de materiales biocompatibles, como polímeros biodegradables y lípidos similares a los componentes de las estructuras celulares (Sahdev y Ochyl., 2014). Las nanopartículas sólidas lipídicas son estructuras similares a las lipoproteínas presentes en el organismo y estas pueden incorporar en su matriz lipídica diversas moléculas y protegerlas de reacciones de degradación que se producen en el medio biológico (Kingwell et al., 2014).

Entre las técnicas que se han empleado para el estudio del metabolismo celular se destaca la espectroscopía de resonancia magnética protónica $(\mathrm{RM}-1 \mathrm{H})$, este constituye un método no invasivo y permite medir el acortamiento del tiempo de relajación espín-espín (T2) que experimentan las moléculas de $\mathrm{H}_{2} \mathrm{O}$ de los hematíes falciformes (Fung et al., 1989). Las variaciones temporales de $\mathrm{T} 2$ describen la cinética de la polimerización de la $\mathrm{HbS}$ intracelular que consta de tres etapas (inicio, polimerización, terminación) y se explica a través del modelo de doble nucleación que consiste en: la nucleación homogénea y heterogénea, ambas coexisten y su efectividad relativa cambia a medida que trascurre proceso. (Menon et al., 1991); (Lester y Bryant 1991).

Considerando lo antes expuesto la investigación tiene como objetivo: determinar la actividad antidedrepanocítica de nanopartículas cargadas con vainillina mediante RMN.

\section{Metodología}

La investigación se desarrolló de conjunto con el Laboratorio de Hematología del Hospital Dr. Antonio María Béguez César y los Laboratorios de Ciencias Biomédicas de la Universidad de Oriente de Santiago de Cuba.

\section{Obtención y purificación de las nanopartículas}

Para la obtención de las nanopartículas se utilizó el método de inyección del disolvente: $50 \mathrm{mg}$ de ácido esteárico (Sigma-Adrich, St. Louis. MO. EE.UU) y 7, $9 \mathrm{mg}$ (concentración requerida para obtener una relación molar hemoglobina-vainillina de 1:2) de vainillina (Sigma-Adrich) fueron disueltos en etanol (Sigma- Adrich) a $80{ }^{\circ} \mathrm{C}$ de temperatura. Esta solución fue colocada en una jeringuilla de $1 \mathrm{~mL}$ e inyectada rápidamente en una solución al 0,1\% del tenso activo Tween 80 (Sigma- Adrich) bajo agitación magnética (IKA - Werke, Staufen, Alemania) a $80{ }^{\circ} \mathrm{C}$. La purificación de la suspensión inicial fue sometida a ultrafiltración utilizando filtros de membranas de 0,450 $\mu \mathrm{m}$. Posteriormente, la solución filtrada fue sometida a centrifugación (Neofuge, Shangai, China) a una velocidad de $16000 \mathrm{rpm}$. Los precipitados fueron re-suspendidos en $1 \mathrm{~mL}$ $\mathrm{PBS} \mathrm{pH}=7.4$ y almacenados a $4{ }^{\circ} \mathrm{C}$ en un refrigerador (Daewoo, Seul, Corea del Sur) (Schubert y Muller, 2003). Para este estudio, fueron preparadas además suspensiones de nanopartículas sólidas lipídicas sin vainillina y disoluciones de este aldehído en las concentraciones adecuadas para el estudio. 


\section{ORANGE JOURNAL}

\section{Determinación de la eficiencia de encapsulación}

La eficiencia de encapsulación de la vainillina se calculó mediante la concentración de esta en los sobrenadantes por método espectrofotométrico, inmediatamente después de la obtención y purificación de las nanopartículas por centrifugación (Castan et al., 2012). Este valor se le restó a la cantidad de vainillina añadida inicialmente y se estimó por diferencia la magnitud de la encapsulación. El método aprovecha la presencia en la estructura de un hidroxilo fenólico y se basa en la reacción de este tipo de compuesto, en medio básico, con el reactivo de Folin Ciocalteu. Debido a esta reacción se desarrolló un color azul, el cual fue leído a $710 \mathrm{~nm}$ en un espectrofotómetro Rayleigh, Taijin, (China). Para la determinación de las concentraciones en las mezclas problemas se realizó una curva de calibración utilizando soluciones patrones de vainillina. El porciento encapsulado se determinó según la ecuacion:

(Ecuación 1)

Donde:

$\% \mathrm{E}=$ porciento de encapsulación

$\mathrm{CVi}=$ concentración de vainillina inicial

CVsn = concentración de vainillina en el sobrenadante

Estudio de la retención de la vainillina por las nanopartículas

La evaluación de la retención de la vainillina en las nanopartículas se realizó según la metodología propuesta por Castan et al., (2012).

$$
\% E=\frac{C V i-C V s n}{C V i} \times 100
$$

\section{Preparación de las Grupos experimentales}

Las muestras biológicas (sangre total de pacientes con AD con hemoglobina SS (SS)) fueron suministradas por el banco de sangre del Hospital Dr. Antonio María Béguez César. Se diseñaron cuatro grupos experimentales y se replicaron tres veces cada una. Grupo 1 (G1): $300 \mu \mathrm{l}$ de sangre total SS y depositándose en un ámpula de RMN para posterior medición.

Grupo 2 (G2): $150 \mu 1$ de sangre total SS y $150 \mu 1$ de formulación de NSL con vainillina y se mezclaron en el ámpula de RMN. Grupo 3 (G3): $150 \mu 1$ de sangre total SS y $150 \mu 1$ de formulación de vainillina y se mezclaron en el ámpula de RMN. Grupo 4 (G4): $150 \mu 1$ de sangre total SS y $150 \mu 1$ de formulación de NSL y se mezclaron en el ámpula de RMN. Las ámpulas fueron selladas con parafilm para asegurar las condiciones anoxigénicas.

Las muestras preparadas fueron medidas en un Relaxómetro GIROMAG 04 (Cuba). Para ello, se utilizaron en todos los grupos experimentales los parámetros provenientes un estándar biológico (para calibración del relaxómetro) relativos al tiempo de demora (Td) 
valor y parámetros anatomofisiológicos ( $\mathrm{pO} 2$ en sangre, $\mathrm{pH}$, concentración de $\mathrm{HbS}$ y de $\mathrm{HbF}$ ) (Fung et al., 1989).

La determinación de la concentración, clasificación, estudio de la cinética de polimerización de la $\mathrm{HbS}$ y el comportamiento temporal de $\mathrm{T} 2$ se realizaron a la frecuencia de $4,4 \mathrm{MHz}$ con la serie de impulsos $90^{\circ}-\tau-180^{\circ}$ según expuesto por Menon et al., (1991) y Lester y Bryant, (1991). Otras condiciones experimentales fueron: 16 promediaciones, 150 pulsos, un tiempo de repetición $(\mathrm{TR})=50000000 \mathrm{~min}$ y un $\mathrm{Tau}=3000$.

\section{Procesamiento estadístico}

Las mediciones de RMN realizadas en el Relaxómetro GIROMAG 04 se ajustaron utilizando el programa Microsoft Excel 2010. Los datos fueron procesados utilizando el software STATISTICA 8.1 para Windows para la comparación de medias entre grupos experimentales a través del empleo del estadígrafo T-student. Se consideró un $95 \%$ de confianza.

\section{Resultados y discusión}

Determinación de la eficiencia de encapsulación e influencia de la concentración de ácido esteárico en la estabilidad de las nanopartículas.

Se obtuvieron nanopartículas sólidas lipídicas con valores de eficiencia de encapsulación superiores al 50\%, lo que demuestra una adecuada incorporación de vainillina en estos nanosistemas.

La Figura 1, muestra que la concentración de ácido esteárico no influye significativamente (según el test estadístico aplicado y con un $95 \%$ de confianza) en la capacidad de retención de la vainillina en las nanopartículas. Ello puede estar relacionado con la liposolubilidad de la vainillina. Este resultado coincide con lo publicado en el 2012 por Castan y colaboradores en donde plantean la eficacia de los liposomas de vainillina dada su capacidad de mantenerse suspendidos en soluciones acuosas y así garantizar su transporte en la sangre (Castan et al., 2012). 


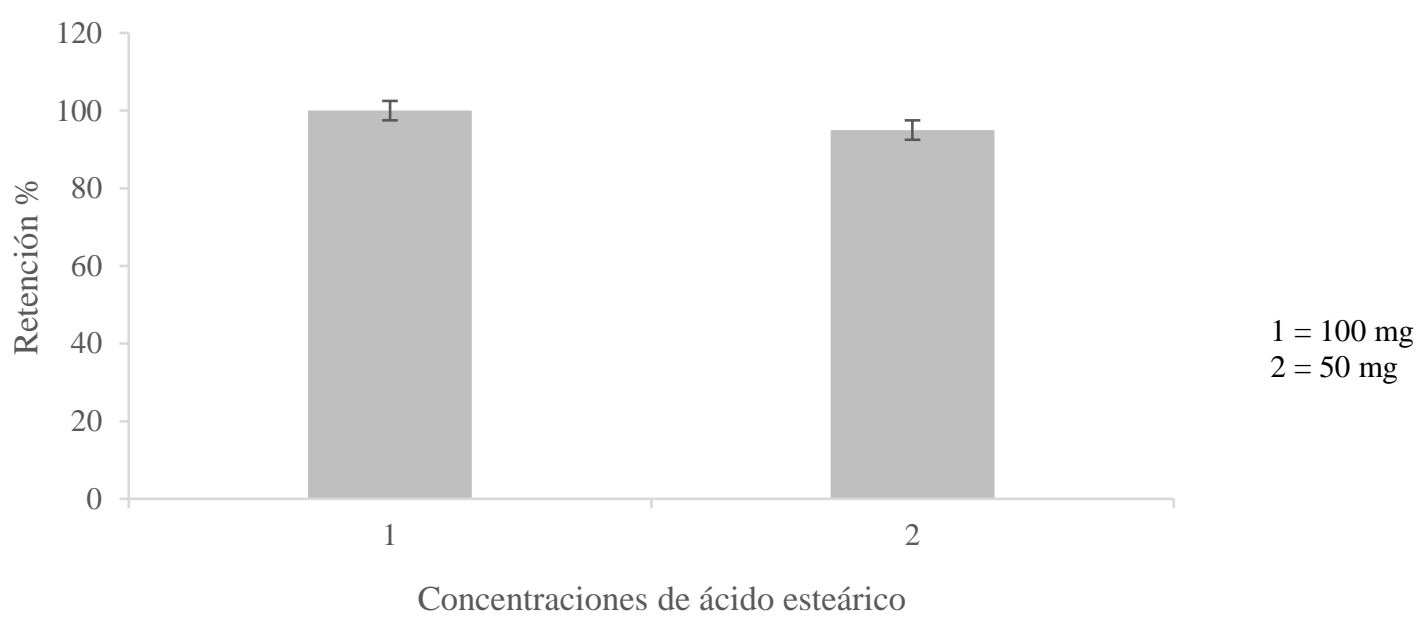

Figura 1. Representación de la capacidad de retención de las nanopartículas para concentraciones de ácido esteárico.

Fuente: Elaborado por los autores.

Estudio de la retención de la vainillina por las nanopartículas

Durante el estudio los valores de Td obtenidos fueron los siguientes valores medios: 120 min para G1, 270 min para G2 y 90 min para los grupos G3 y G4, observándose similitud para los grupos G3 y G4. Ello es una muestra del incremento significativo para el tratamiento con nanopartículas sólidas lipídicas cargadas con vainillina (NSL-V).

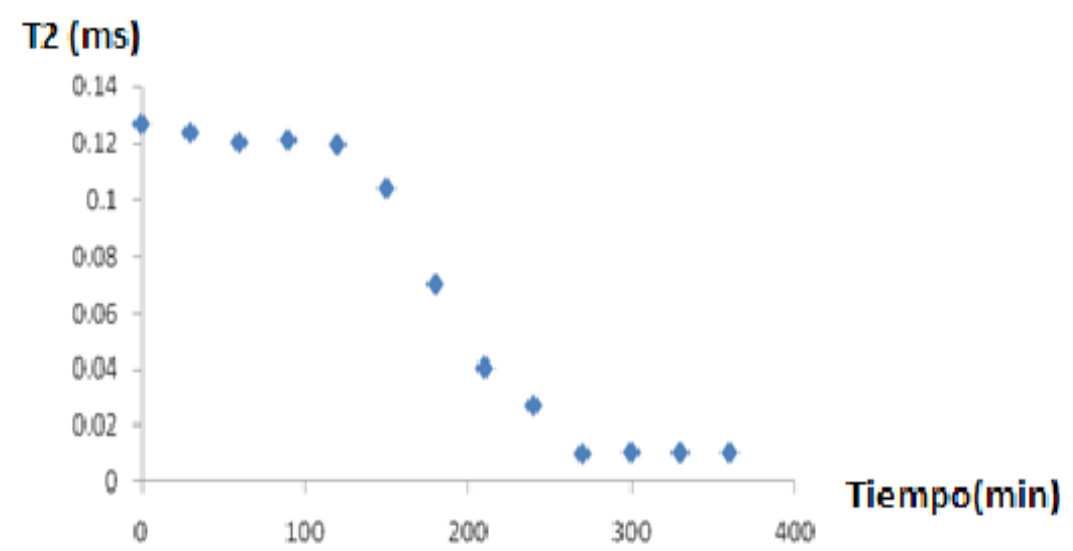

Figura 2. Grupo 1 (Sangre total SS) 


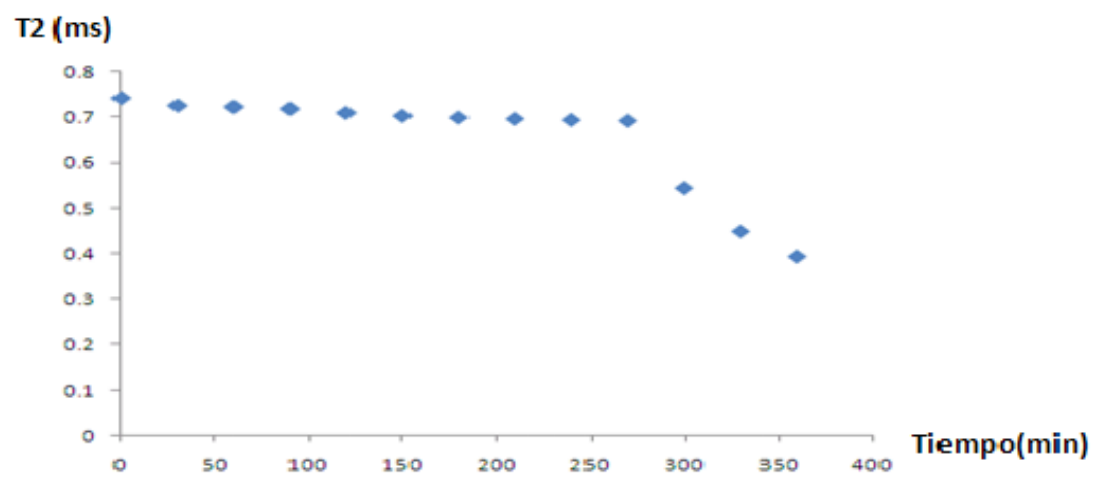

Figura 3. Grupo 2 (Sangre SS + NSLV)

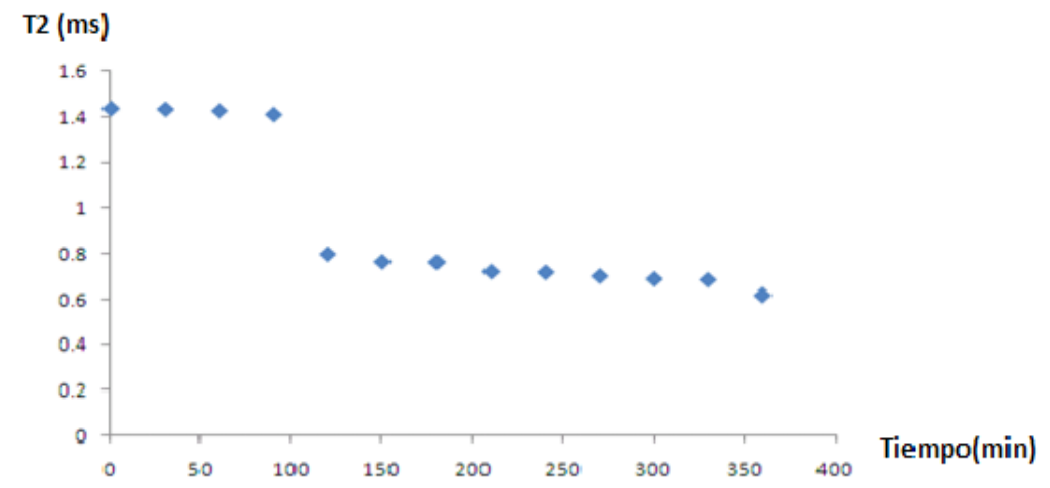

Figura 4. Grupo 3 (Sangre SS + vainillina)

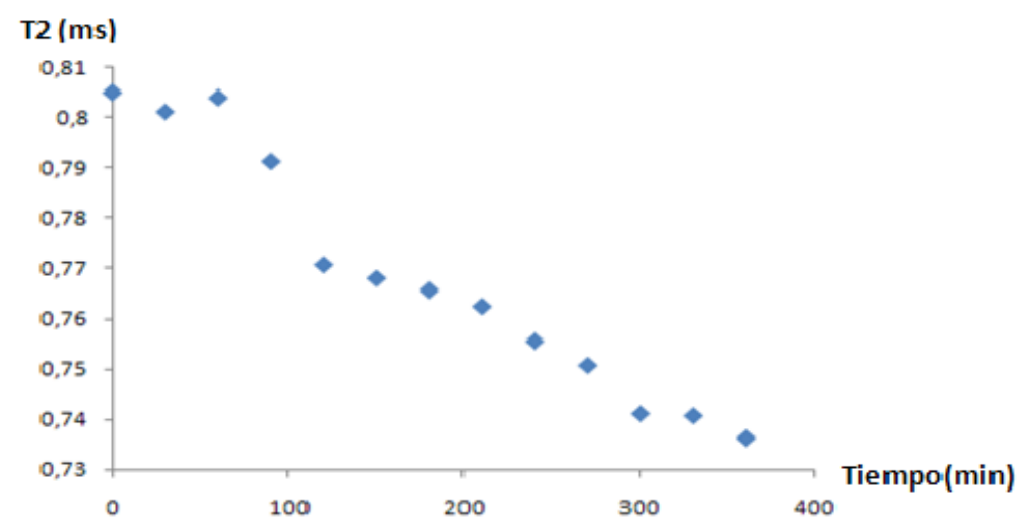

Figura 5. Grupo 4 (Sangre SS + NSL)

Fuente: Elaborado por los autores. 


\section{ORANGE JOURNAL}

La formulación correspondiente al grupo experimental, G1 (300 $\mu 1$ de sangre total SS) describió la curva sigmoidea característica de los procesos de polimerización que ocurren en los pacientes con AD (Figura 2). El aumento de los valores del T2 y el alargamiento del Td de la polimerización es una consecuencia de la movilidad de las moléculas de agua por inhibición del polímero (Strader et al., 2019), (Maruyama et al., 2020).

El grupo experimental G2 ( $150 \mu 1$ de sangre total SS y $150 \mu 1$ de formulación de NSL con vainillina muestra el mayor Td (Figura 3). Esto puede estar condicionado a que las altas concentraciones intracelulares de vainillina establezcan un equilibrio entre las moléculas de esta sustancia libres en el medio y las enlazadas a la HbS. Esto podría explicar el efecto retardador lo que sugiere una saturación de los sitios de unión entre $\mathrm{Hb}$ - vainillina y una mayor efectividad en la ralentización del proceso de polimerización (Wood et al., 2014), (Deshpande et al., 2018), (Gour et al., 2020).

Los grupos experimentales G3 $(150 \mu 1$ de sangre total SS y $150 \mu 1$ de formulación de NSL) y G4 (150 $\mu$ l de sangre total SS y $150 \mu$ l de formulación de NSL) representados por las figuras 4 y 5 respectivamente mostraron iguales valores de $\mathrm{Td}$, a pesar de ser formulaciones diferentes. Esto puede deberse a que las moléculas de vainillina en el torrente sanguíneo interaccionan con otros componentes del tejido sangre en vez de atravesar la membrana celular de los eritrocitos, la cual supone una barrera importante para muchos fármacos (Chen et al., 2017).

Al comprar los resultados obtenidos con los reportados por García et al., (2005), y Torres et al., (2005) se observó que los tiempos de demora fueron mucho menores. Esta diferencia puede estar relacionada a dos factores: la influencia de la membrana en la señal de RMN, y la presencia en sangre de moléculas moduladoras de la afinidad de la $\mathrm{Hb}$ por el oxígeno, como es el caso del 2,3 bifosfoglicerato (2, 3 BPG) (Sarkar et al., 2017).

La membrana celular de los eritrocitos influye en la magnitud de los tiempos de demora. Factores como la permeabilidad de la membrana celular pueden influir en la movilidad del agua, ya que las membranas biológicas son semipermeables y pueden regular la entrada y salida de diversas sustancias. En el caso de los eritrocitos con $\mathrm{HbS}$, se ha comprobado que estos pueden presentar alteraciones en la permeabilidad, lo que afecta el movimiento de las moléculas de agua. Las alteraciones presentes en las membranas de los eritrocitos falciformes pueden traer como consecuencia una deshidratación rápida y severa de los glóbulos rojos (Gallagher, 2017).

Estos cambios en el contenido de agua pueden afectar los valores de Td en sangre total al ser comparados con soluciones de $\mathrm{HbS}$, en donde la movilidad de las moléculas de agua no depende de la permeabilidad de las membranas biológicas. Como consecuencia de lo anterior, puede asumirse que en el caso de los eritrocitos SS, la elevada tasa de polimerización de la $\mathrm{HbS}$ y la rápida deshidratación provoca un aumento del movimiento de las moléculas de agua causando que todo el proceso seguido por RMN se registre en menos tiempo, por lo que la curva se desplaza hacia la izquierda y el Td disminuye (Brugnara, 2018).

El segundo factor que debe ser considerado es la concentración de agentes moduladores 
de la afinidad de la $\mathrm{Hb}$ por el oxígeno como el 2, 3 BPG. Esta molécula es capaz de unirse a la $\mathrm{Hb}$ y disminuir la afinidad de esta por el oxígeno. Fisiológicamente el 2, 3 BPG permite que el oxígeno sea liberado hacia los tejidos, ya que de lo contrario este permanecería retenido en las moléculas de $\mathrm{Hb}$. El 2,3- BPG se enlaza al centro del tetrámero de $\mathrm{Hb}$, en un 'bolsillo' que sólo está presente en el estado tenso o de menor afinidad por el oxígeno de esta molécula (T). En la transición de la molécula $\mathrm{T}$ al estado relajado o estado de mayor afinidad por el oxígeno (R), se libera el 2,3- BPG por colapso del 'bolsillo'. La ruptura de los enlaces entre la hemoglobina y el 2,3- BPG provocará que se cambie del estado $\mathrm{T}$ al $\mathrm{R}$, condicionando el incremento del número de enlaces de oxígeno con la hemoglobina. Es por ello que la hemoglobina se mantiene en su estado $\mathrm{T}$ hasta que un medio con altas concentraciones de oxígeno condicione su cambio. Esta propiedad del 2,3- BPG es conocida como efecto alostérico, que no es más que la "regulación" de la unión de las moléculas de oxígeno con la hemoglobina desde su unión a un sitio estructuralmente diferente al del O2 (Nelson y Cox, 2019), (Ghysels et al., 2019).

Se ha demostrado que en un estado puro la hemoglobina se enlaza fuertemente al oxígeno, dificultando su liberación, mientras que en las células rojas su afinidad es menor. Esta diferencia se debe a la presencia del 2,3- BPG en las células rojas de la sangre a una concentración elevada, mientras que en la hemoglobina purificada o en solución, la concentración es baja. Al existir bajas concentraciones de 2,3- BPG en las soluciones de $\mathrm{HbS}$, las curvas sigmoideas resultantes de los experimentos donde están presentes estas soluciones deben estar desplazadas hacia la derecha, lo que demuestra una mayor afinidad por el oxígeno en dichos casos. Lo anterior conlleva a que todo el proceso seguido por RMN sea más lento y se obtengan mayores valores de Td, en comparación con la sangre total, en donde la concentración de 2, 3 BPG es mucho mayor (Oslund et al., 2017).

El hecho de que la vainillina en nanopartículas haya resultado superior en retardar la polimerización, corrobora lo que ha sido demostrado en otros estudios con nanopartículas para el aumento de la penetración intracelular de moléculas activas biológicamente (García et al., 2005). La presente investigación aporta un dato relevante sobre el comportamiento de la vainillina en sangre total, al demostrar que su capacidad de inhibir la polimerización de la $\mathrm{HbS}$.

\section{Conclusiones}

Se obtuvieron nanopartículas sólidas lipídicas con valores de eficiencia de encapsulación superiores al 50\%, lo que demuestra una adecuada incorporación de vainillina en estos nanosistemas. Mediante el empleo de la técnica RMN se demostró que la vainillina encapsulada en NSL aumenta los valores de Td, lo que es indicativo de la inhibición de la formación de los polímeros de $\mathrm{HbS}$.

\section{Referencias bibliográficas}

Beddell, C. R., Kneen, G., \& White, R. D. (1979). The anti-sickling activity of a series of aromatic aldehydes (proceedings). British journal of pharmacology, 66(1), p. 70. 


\section{ORANGE JOURNAL}

Brugnara, C. (2018). Sickle cell dehydration: Pathophysiology and therapeutic applications. Clinical hemorheology and microcirculation, 68(2-3), 187-204.

Cabal, C., Fernández, A., Lores, M., Álvarez, E., Losada, J., Soler, C. \& Pérez, E. (1998). Magnetic relaxation in the kinetics of the polymerization of hemoglobin S. Clinical diagnosis and treatment with vanillin. In Proceedings International Society for Magnetic Resonance in Medicine, Vol. 3, p. 1705.

Castan, C, L., del Toro, G. G., Fernández, G, A. A., González P, M., Ortiz B, E., \& Lobo T, D. (2012). Encapsulación del 4-Hidroxi-3-metoxibenzaldehído en liposomas modificados con 1-O-alquilgliceroles sintéticos: estudio de su reactividad con el radical DPPH. Revista Cubana de Química, 24(1), 83-90.

Chen, W. R., Yu, Y., Zulfajri, M., Lin, P. C., \& Wang, C. C. (2017). Phthalide derivatives from Angelica Sinensis decrease hemoglobin oxygen affinity: a new allosteric-modulating mechanism and potential use as 2, 3-BPG functional substitutes. Scientific reports, 7(1), 1-15.

Deshpande, T. M., Pagare, P. P., Ghatge, M. S., Chen, Q., Musayev, F. N., Venitz, J. \& Safo, M. K. (2018). Rational modification of vanillin derivatives to stereospecifically destabilize sickle hemoglobin polymer formation. Acta Crystallographica Section D: Structural Biology, 74(10), 956-964.

Fung, L. M., Narasimhan, C., Lu, H. Z., \& Westerman, M. P. (1989). Reduced water exchange in sickle cell anemia red cells: a membrane abnormality. Biochimica et Biophysica Acta (BBA)-Biomembranes, 982(1), 167-172.

Gallagher, P. G. (2017). Disorders of erythrocyte hydration. Blood. The Journal of the American Society of Hematology, 130(25), 2699-2708.

García, A. F., Cabal, C., Losada, J., Álvarez, E., Soler, C., \& Otero, J. (2005). In vivo action of Vanillin on delay time determined by magnetic relaxation. Hemoglobin, 29(3), 181-187.

Ghysels, A., Krämer, A., Venable, R. M., Teague, W. E., Lyman, E., Gawrisch, K., \& Pastor, R. W. (2019). Permeability of membranes in the liquid ordered and liquid disordered phases. Nature communications, 10(1), 1-12.

Gour, A., Dogra, A., Bhatt, S., \& Nandi, U. (2020). Effect of Natural Products on Improvement of Blood Pathophysiology for Management of Sickle Cell Anemia. Botanical Leads for Drug Discovery (pp. 51-65). Springer, Singapore.

Kingwell, B. A., Chapman, M. J., Kontush, A., \& Miller, N. E. (2014). HDLtargeted therapies: progress, failures and future. Nature reviews Drug discovery, 13(6), 445-464.

Lester, C. C., \& Bryant, R. G. (1991). Water-proton nuclear magnetic relaxation in heterogeneous systems: Hydrated lysozyme results. Magnetic resonance in medicine, 22(1), 143-153.

Lu, C. T., Zhao, Y. Z., Wong, H. L., Cai, J., Peng, L., \& Tian, X. Q. (2014). Current approaches to enhance CNS delivery of drugs across the brain barriers. International Journal of Nanomedicine, 9, 2241.

Maruyama, T., Fukata, M., \& Fujino, T. (2020). Physiological and pathophysiological significance of erythrocyte senescence, density and deformability: Important but unnoticed trinity. Journal of Biorheology, 34(2), 61-70

Menon, R. S., Rusinko, M. S., \& Allen, P. S. (1991). Multiexponential proton relaxation in model cellular systems. Magnetic resonance in medicine, 20(2), 196-213. 
Morejón, L. D., Jorge, B. R., Sánchez, D. G., Rayas, Y. L., Lezcano, L. A., \& Leonard, M. E. S. (2019). Anemia drepanocítica: características generales de los pacientes a su diagnóstico. Revista de Enfermedades no Transmisibles, 9(1), 4-10.

Nelson, D, L., Cox, M. (2019) Lehninger. Principles of Biochemistry. 7a ed. 1260p. Ed. W.H Freeman \& Co. Ltd.

Nigen, A. M., \& Manning, J. M. (1977). Inhibition of erythrocyte sickling in vitro by DL-glyceraldehyde. Proceedings of the National Academy of Sciences, 74(1), 367-371.

Oslund, R. C., Su, X., Haugbro, M., Kee, J. M., Esposito, M., David, Y. \& Rabinowitz, J. D. (2017). Bisphosphoglycerate mutase controls serine pathway flux via 3-phosphoglycerate. Nature chemical biology, 13(10), 1081.

Sahdev, P., Ochyl, L. J., \& Moon, J. J. (2014). Biomaterials for nanoparticle vaccine delivery systems. Pharmaceutical research, 31(10), 2563-2582.

Sarkar, H. S., Das, S., Uddin, M. R., Mandal, S., \& Sahoo, P. (2017). Selective Recognition and Quantification of 2, 3-Bisphosphoglycerate in Human Blood Samples by a Rhodamine Derivative. Asian Journal of Organic Chemistry, 6(1), 71-75.

Schubert, M. A., \& Müller-Goymann, C. C. (2003). Solvent injection as a new approach for manufacturing lipid nanoparticles-evaluation of the method and process parameters. European journal of pharmaceutics and biopharmaceutics, 55(1), 125-131.

Strader, M. B., Liang, H., Meng, F., Harper, J., Ostrowski, D. A., Henry, E. R. \& Alayash, A. I. (2019). Interactions of an anti-sickling drug with hemoglobin in red blood cells from a patient with sickle cell anemia. Bioconjugate chemistry, 30(3), 568-571.

Torres, D, A., del Toro, G, G., Valdés, R, Y. C., León, J. L., \& Merchán, F. (2005). Effect in vitro of synthetic 1-O-alkylglycerols on the transformation and hemolytic activity on the sickle erythrocytes. Bioquimia, 30(4), 101-109.

Wood, A. R., Esko, T., Yang, J., Vedantam, S., Pers, T. H., Gustafsson, S. \& Lichtner, P. (2014). Defining the role of common variation in the genomic and biological architecture of adult human height. Nature genetics, 46(11), 1173-1186. 